\title{
Continuous harvest of stem cells via partial detachment from thermoresponsive nanobrush surfaces
}

\begin{abstract}
Stem cell culture is typically based on batch-type culture, which is laborious and expensive. Here, we propose a continuous harvest method for stem cells cultured on thermoresponsive nanobrush surfaces. In this method, stem cells are partially detached from the nanobrush surface by reducing the temperature of the culture medium below the critical solution temperature needed for thermoresponse. The detached stem cells are harvested by exchange into fresh culture medium. Following this, the remaining cells are continuously cultured by expansion in fresh culture medium at $37{ }^{\circ} \mathrm{C}$. Thermoresponsive nanobrush surfaces were prepared by coating block copolymers containing polystyrene (for hydrophobic anchoring onto culture dishes) with three types of polymers: (a) polyacrylic acid with cell-binding oligopeptides, (b) thermoresponsive poly-N-isopropylacrylamide, and (c) hydrophilic poly(ethyleneglycol)methacrylate. The optimal coating durations and compositions for these copolymers to facilitate adequate attachment and detachment of human adipose-derived stem cells (hADSCs) and embryonic stem cells (hESCs) were determined. hADSCs and hESCs were continuously harvested for 5 and 3 cycles, respectively, via the partial detachment of cells from thermoresponsive nanobrush surfaces.
\end{abstract}

Keyword: Thermoresponsive; Stem cell; Nanobrush; Cell culture; Embryonic stem cell; Biomaterial 